\title{
IW And-Type State in IM Eridani
}

Taichi Kato, ${ }^{1 *}$ Yasuyuki WaKamatsu, ${ }^{1}$ Naoto KoJIGuchI, ${ }^{1}$ Mariko KImUrA, ${ }^{1}$ Ryuhei OHNISHI, ${ }^{1}$ Keisuke IsOgal, ${ }^{1,2}$ Keito NiIJImA, ${ }^{1}$ Tomohiro Yoshitake, ${ }^{1}$ Yuki SugIURA, ${ }^{3}$ Sho SumiYA, ${ }^{3}$ Daiki Ito, ${ }^{3}$ Kengo NiKal, ${ }^{3}$ Hanami Matsumoto, ${ }^{3}$ Katsura Matsumoto, ${ }^{3}$ Tonny Vanmunster, ${ }^{4}$ Franz-Josef HambSCH, ${ }^{5,6,7}$ Hiroshi ITOH, ${ }^{8}$ Julia V. Babina, ${ }^{9}$ Oksana I. AntonyuK, ${ }^{9}$ Alex V. BaKlanov, ${ }^{9}$ Elena P. Pavlenko, ${ }^{9}$ Berto Monard, ${ }^{10,11}$ Shawn DvoraK, ${ }^{12}$

${ }^{1}$ Department of Astronomy, Kyoto University, Kyoto 606-8502, Japan

2 Okayama Observatory, Kyoto University, 3037-5 Honjo, Kamogatacho, Asakuchi, Okayama 719-0232, Japan

3 Osaka Kyoiku University, 4-698-1 Asahigaoka, Osaka 582-8582, Japan

${ }^{4}$ Center for Backyard Astrophysics Belgium, Walhostraat 1A, B-3401 Landen, Belgium

${ }^{5}$ Groupe Européen d'Observations Stellaires (GEOS), 23 Parc de Levesville, 28300 Bailleau l'Evêque, France

${ }^{6}$ Bundesdeutsche Arbeitsgemeinschaft für Veränderliche Sterne (BAV), Munsterdamm 90, 12169 Berlin, Germany

7 Vereniging Voor Sterrenkunde (VVS), Oude Bleken 12, 2400 Mol, Belgium

8 Variable Star Observers League in Japan (VSOLJ), 1001-105 Nishiterakata, Hachioji, Tokyo 192-0153, Japan

9 Federal State Budget Scientific Institution "Crimean Astrophysical Observatory of RAS", Nauchny, 298409, Republic of Crimea

10 Bronberg Observatory, Center for Backyard Astrophysics Pretoria, PO Box 11426, Tiegerpoort 0056, South Africa

11 Kleinkaroo Observatory, Center for Backyard Astrophysics Kleinkaroo, Sint Helena 1B, PO Box 281, Calitzdorp 6660, South Africa

12 Rolling Hills Observatory, 1643 Nightfall Drive, Clermont, Florida 34711, USA

*E-mail: *tkato@kusastro.kyoto-u.ac.jp

Received 201 0; Accepted 2010

\section{Abstract}

IW And stars are a recently recognized group of dwarf novae which are characterized by a repeated sequence of brightening from a standstill-like phase with damping oscillations followed by a deep dip. Kimura et al. (2019) recently proposed a model based on thermal-viscous disk instability in a tilted disk to reproduce the IW And-type characteristics. IM Eri experienced the IW And-type phase in 2018 and we recorded three cycles of the (damping) oscillation phase terminated by brightening. We identified two periods during the IW And-type state: $4-5 \mathrm{~d}$ small-amplitude (often damping) oscillations and a 34-43 d long cycle. This behavior is typical for an IW And-type star. The object gradually brightened within the long cycle before the next brightening which terminated the (damping) oscillation phase. This observation agrees with the increasing disk mass during the long cycle predicted by a model of thermal-viscous disk instability in a tilted disk (Kimura et al. 2019). We, however, did not succeed in detecting negative superhumps, which are considered to be the signature of a tilted disk.

(C) 2014. Astronomical Society of Japan. 
Key words: accretion, accretion disks - stars: novae, cataclysmic variables - stars: dwarf novae stars: individual (IM Eridani)

\section{Introduction}

Cataclysmic variables (CVs) are binary stars consisting of a white dwarf and a mass-transferring secondary filling the Roche lobe. The transferred matter forms an accretion disk around the white dwarf. Some CVs show outbursts with amplitudes of several magnitudes and they are called dwarf novae (DNe). Some CVs do not show prominent outbursts and stay at almost constant brightness. They are usually called novalike variables (NLs). It has been demonstrated that thermal instability in the accretion disk causes outbursts in DNe [see e.g. Osaki (1996); for general information of cataclysmic variables and dwarf novae, see e.g. Warner (1995)]. In this diskinstability model, objects with sufficiently high masstransfer rates $(\dot{M})$ have thermally stable disks and they are considered to explain NLs without prominent outbursts.

Some CVs lie near the border of DNe and NLs. The best-known class is Z Cam stars, which at times show DNtype outbursts and also NL-type quasi-constant phases called standstills. It is widely believed that changing $\dot{M}$ from the secondary causes both DN and NL states in the same object depending on $\dot{M}$ (Meyer and MeyerHofmeister 1983).

Quite recently, another class of objects (also regarded as a subclass of $Z$ Cam stars) has been recognized. The initial two objects, IW And and V513 Cas, showed similar light curves characterized by brightening from a standstill (or standstill-like phase) followed by a deep dip. These features are known to recur quasi-periodically (Simonsen 2011). These two stars were studied in detail by Szkody et al. (2013). Hameury and Lasota (2014) called these objects "anomalous Z Cam stars" and suggested that brightening from a standstill and a following dip are caused by an enhancement and a subsequent decay of $\dot{M}$ from the secondary. Following Hameury and Lasota (2014), these enhancements and decays of $\dot{M}$ need to occur repeatedly to explain the behavior of these objects. Hameury and Lasota (2014) described that "these [mass-transfer] outbursts are not found in most systems and may thus be due to some rare set of circumstances/parameters" and admitted the difficulty within the current knowledge.

Kato (2019) identified three more objects V507 Cyg, IM Eri and FY Vul showing the similar behavior. Adding two more objects in the literature [ST Cha (Simonsen et al. 2014); KIC 9406652 (Gies et al. 2013)], Kato (2019) defined the class having properties of a sequence of a standstill terminated by brightening followed by damping oscillations. They are now widely referred to as IW And-type stars following the suggestion by Kato (2019). There was yet another object in the literature [V523 Lyr (Mason and Howell 2016)] which was not included in Kato (2019).

The key difference of IW And-type objects from the classical Z Cam stars can be summarized as follows: (1) Standstills are terminated by brightening in IW And-type objects, unlike fading in Z Cam stars. The termination of standstills in Z Cam stars is usually understood as a consequence of the decreasing $\dot{M}$ from the secondary [see e.g. Warner (1995)] and brightening at the end of a standstill is not expected from this picture. (2) In IW Andtype objects, there are quasi-periodic cycles consisting of a (quasi-)standstill with damping oscillations - brightening which terminates the standstill — often a deep dip (not always present within the same object) and returning to a (quasi-)standstill. Standstills in Z Cam stars occur irregularly and no such cycles are known.

Kato (2019) suggested the presence of a previously unknown type of limit-cycle oscillation to reproduce the cycle. They proposed that a (quasi-)standstill in these objects are somehow maintained in the inner part of the disk and that thermal instability starting from the outer part of the disk sweeps the disk, thereby terminating the standstill to complete the cycle. The idea of a limit cycle is advantageous in the absence of a known mechanism to produce recurring mass-transfer outbursts (Hameury and Lasota 2014).

In line with this suggestion and using the evidence of a tilted disk in KIC 9406652 (Gies et al. 2013), Kimura et al. (2019) proposed an idea that the transferred matter can reach the inner portion of a tilted disk. The transferred matter to the inner disk thermally stabilizes the inner disk, which was expected to reproduce (quasi-)standstills in IW And-type objects. Using a one-dimensional thermal-viscous disk instability model, Kimura et al. (2019) were able to reproduce a limit-cycle oscillation consisting of standstill-like damping oscillations terminated by brightening. This limit cycle reflects the secular increase of the disk mass during standstill-like damping oscillations, which is swept by the cooling wave caused by thermal instability from the outer disk. This limit cycle is analogous to the one in SU UMa-type dwarf 
novae, in which the secular increase of the angular momentum plays a similar role (Osaki 1996). The existence of a cycle and brightening terminating the (quasi-)standstill are two key elements of the IW And-type phenomenon.

\section{IM Eridani}

Among newly identified IW And-type stars in Kato (2019), IM Eri had been known as an NL variable (Chen et al. 2001; Armstrong et al. 2013). Armstrong et al. (2013) spectroscopically identified the orbital period to be $0.1456346(2) \mathrm{d}$ and found strong negative superhumps with a period of 0.13841(3) d in their 2002 and 2012 observations. Negative superhumps are semi-periodic variations having periods slightly shorter than the orbital period and are considered to arise from a precessing tilted disk (cf. Wood and Burke 2007; Montgomery 2009). According to the All Sky Automated Survey (ASAS-3) observations (Pojmański 2002), this object was in the NL state between 2000 November and 2005 April, but apparently showed outbursts between 2005 May and 2009 December. There were some time-series observations in the AAVSO database ${ }^{1}$ between 2011 December and 2016 March, all showing NL states. Most recently, the All Sky Automated Survey for Supernovae (ASAS-SN) Sky Patrol (Shappee et al. 2014; Kochanek et al. 2017) started observations since 2013 October, which eventually led to an identification as an IW And-type star starting from the dwarf nova-type state since 2016 September. Although some of this dwarf nova-type state was covered by the AAVSO database in 2016-2017, the data were too sparse to clarify the IW And-type nature.

After the identification of the ongoing IW And-type state in IM Eri in 2018 October, we initiated a campaign. According to Kimura et al. (2019), even the same object can display a variety of light variations depending on the tilt angle of the disk. The IW And-type state in IM Eri was expected to provide an excellent opportunity to study the relationship between the disk tilt and light variation.

\section{Observation and Analysis}

The data were acquired by the contributors to the VSNET Collaboration (Kato et al. 2004) with $20-60 \mathrm{~cm}$ telescopes located world-wide. All observers used $V$-filtered or unfiltered CCD cameras. They used aperture photometry and extracted magnitudes relative to comparison stars whose constancy has been confirmed by comparison with check stars. The small differences between observers were corrected by adding constants to minimize the squared

$1<$ http://www.aavso.org/data-download $>$. sum of adjacent observations in the combined light curve. The times of all observations are expressed in barycentric Julian days (BJD). We mainly used $\mathrm{R}$ software ${ }^{2}$ for data analysis. The log of observations and corrections to individual observers are listed in E-table 1.

We used the phase dispersion minimization (PDM, Stellingwerf 1978) method to identify periods in the data. The error of the PDM analysis was estimated by the methods of Fernie (1989) and Kato et al. (2010). Although we also applied the least absolute shrinkage and selection operator (Lasso) method (Tibshirani 1996; Kato and Uemura 2012) particularly using the two-dimensional version (Kato and Maehara 2013), we do show the result since we could not find particular advantage over the PDM method.

\section{Results}

\subsection{Long-term behavior}

The resultant light curve is shown in figure 1. Our observation started just after the "terminal outburst" (TO0), which was recorded by ASAS-SN. The object showed oscillations and gradually brightened. This oscillation phase was terminated by the outburst TO1. We observed three terminal outbursts $\mathrm{TO} 1, \mathrm{TO} 2$ and $\mathrm{TO} 3$ during our campaign. The intervals between them were $43 \mathrm{~d}$ and $41 \mathrm{~d}$. The interval between TO0 and TO1 was $34 \mathrm{~d}$, suggesting that the intervals were not perfectly regular.

Although the oscillations between TO0 and TO1 were not particularly damping, they were clearly damping between TO1 and TO2. In the segment between TO2 and TO3, the oscillations were clearly seen with small amplitudes only for the initial two cycles and gradual brightening toward TO3 remained after them. The periods of oscillations were 4.34(3) d in TO0-TO1 and 5.05(3) d in TO1-TO2. These periods were also not constant.

\subsection{Orbital and superhump variations}

We show the results of the PDM analysis of (damping) oscillation phases (including TO1) before TO2 in figure 2 . To remove the trend of oscillations, the original data were cut to 1-d segments and de-trended using the locallyweighted polynomial regression (LOWESS: Cleveland 1979) when the data were abundant, and using a linear regression when the data number of each bin was less than 50. The result was virtually the same when we treated longer segments by LOWESS only. The result was also virtually the same when we included/excluded terminal

\footnotetext{
2 The R Foundation for Statistical Computing:

$<$ http://cran.r-project.org/>.
} 


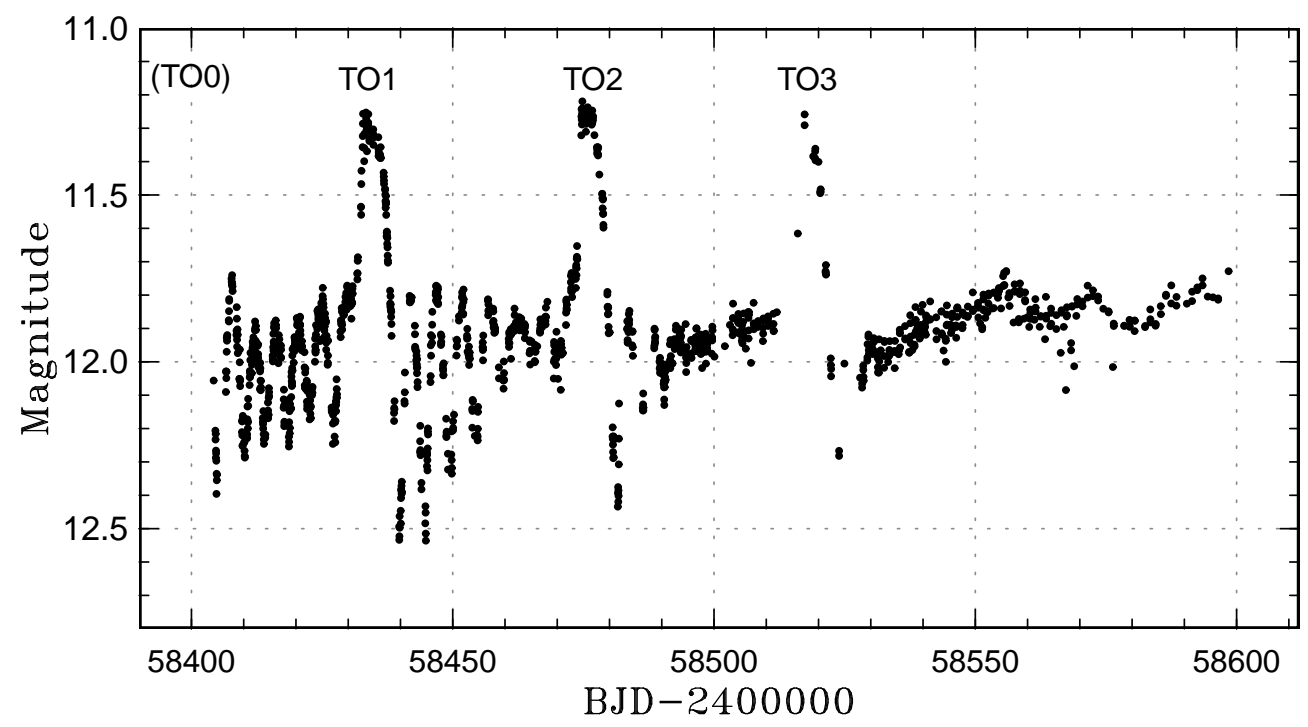

Fig. 1. Light curve of IM Eri in 2018 from our CCD observations. The data were averaged to 0.03 d bins. We observed three "terminal outbursts" (TO1, TO2, TO3) at the end of damping oscillations. The earlier epoch of a terminal outburst is shown as "(TO0)", which was recorded by ASAS-SN, but the data were insufficient to depict variations before and after it.

outbursts in the segment of analysis. No signal was detected between $\mathrm{TO} 2$ and $\mathrm{TO} 3$, probably due to the smaller number of observations.

The data showed only a weak signal of the orbital period at $0.1456(1) \mathrm{d}$, which is identical with the spectroscopic period of 0.1456346(2) d in Armstrong et al. (2013). There was no signal of negative superhumps near the expected region around $0.1384 \mathrm{~d}$. To check the effect of detrending, we also performed the PDM analysis without de-trending or weaker de-trending by changing LOWESS parameters. No signal of negative superhumps appeared regardless of the degree of de-trending. We also applied analysis for shorter segments (E-figures 1,2) without detectable signal of negative superhumps. We conclude that the amplitude of negative superhumps was below the detection limit (0.01 mag).

The result was very different from the PDM analysis of the 2012 data [a major part of the data used in Armstrong et al. (2013) is available in the AAVSO database] when the object was in the NL state and negative superhumps were very strong (amplitude of $0.16 \mathrm{mag}$, figure 3 ).

\section{Discussion}

\subsection{Overall behavior}

We found that IM Eri showed two periods during the IW And-type state: 4-5 d small-amplitude oscillations (often damping) and a 34-43 d long cycle. This behavior is typical for an IW And-type star.
We first examine whether these small-amplitude oscillations reflected the intrinsic variation of the disk luminosity or whether they were caused by the varying aspect of a precessing tilted disk. Even though there remains a possibility of a tilted disk producing undetectably weak negative superhumps, the beat period between the orbital period and the period of recorded negative superhumps in IM Eri is $2.8 \mathrm{~d}$, which cannot explain the 4-5 d smallamplitude oscillations. The small-amplitude oscillations thus should arise from the intrinsic variation of the disk luminosity.

In the case of the Kepler object KIC 9406652, the longterm variation was strongly affected by the beat period between the orbital period and the period of negative superhumps (Gies et al. 2013) and it is difficult to unambiguously distinguish damping oscillations and the beat variation (the full treatment of this issue considering the variable precession rate will be discussed in Kimura et al. in prep.). Since the orbital periods and periods of negative superhumps, if present, were unknown or only poorly known in other IW And-type stars, the distinction between damping oscillations and the beat phenomenon is even more difficult. IM Eri thus provides the first clear demonstration of the (damping) oscillations between terminal outbursts by time-resolved photometry.

There was a gradual brightening tendency during the (damping) oscillation phase. This gradual brightening cannot be explained straightforwardly by recurrent mass-transfer bursts as proposed by Hameury and Lasota (2014). This tendency, however, can be well explained by 

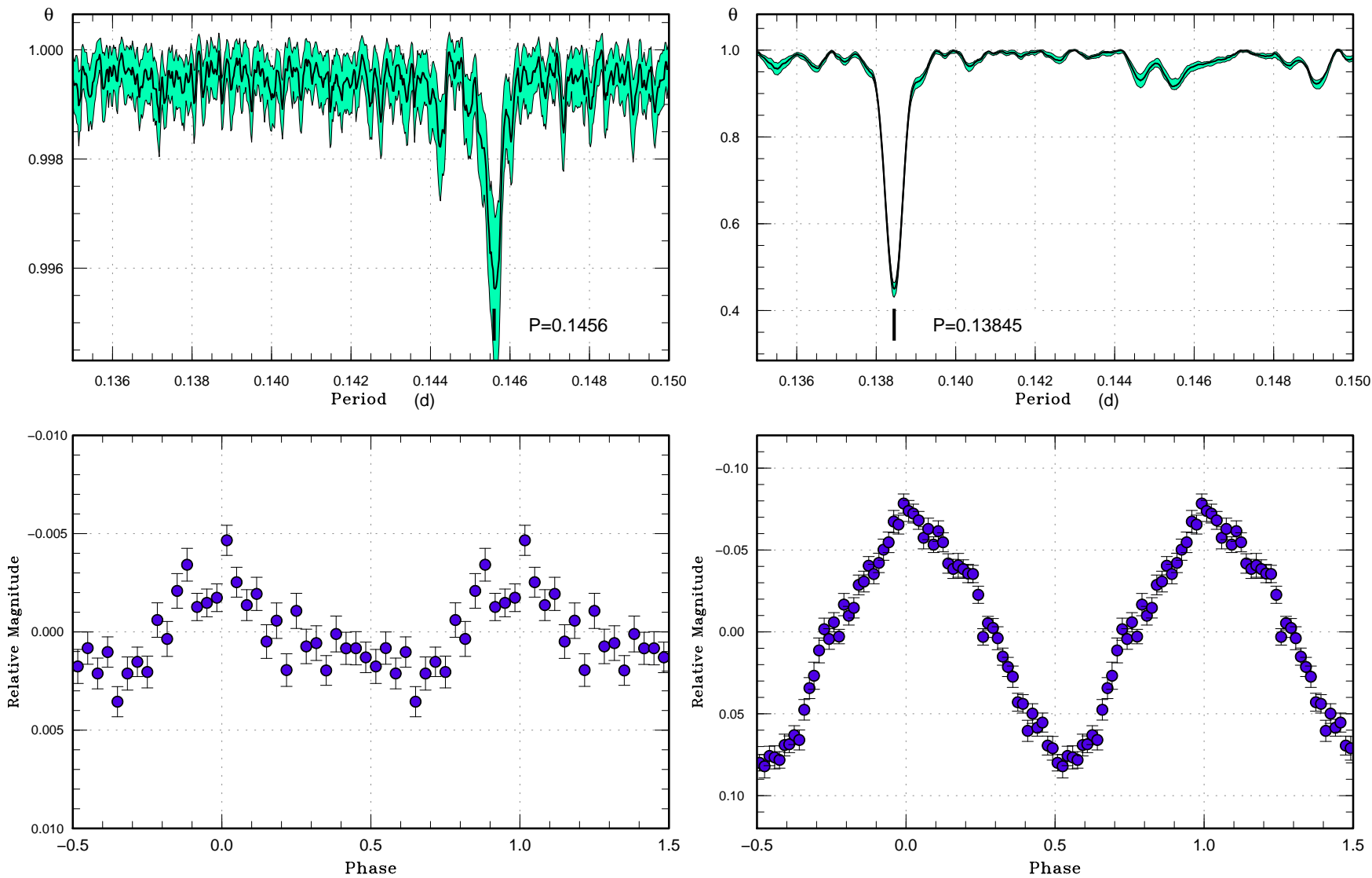

Fig. 2. PDM analysis of IM Eri before BJD 2458474 (before TO2). We analyzed 100 samples which randomly contain $50 \%$ of observations, and performed the PDM analysis for these samples. The bootstrap result is shown as a form of $90 \%$ confidence intervals in the resultant PDM $\theta$ statistics.

Fig. 3. PDM analysis of IM Eri in the NL state in 2012. Negative superhumps were very strong. The orbital signal at $0.1456 \mathrm{~d}$ was much weaker than the signal of negative superhumps.

the gradual increase of the disk mass by the model of a tilted disk by Kimura et al. (2019).

After TO3, this object gradually brightened without oscillations. (Terminal) outbursts also disappeared. It was likely that $\dot{M}$ gradually increased after TO2: the outer part of the disk gradually became thermally stable, which suppressed thermal instability producing a terminal outburst to occur.

\subsection{Evidence for disk tilt}

In contrast to the 2012 observations, IM Eri did not show a strong sign of negative superhumps, which is the signature of a disk tilt, during the IW And-type state. This is against what would be expected by Kimura et al. (2019). The 2012 NL state was probably indeed achieved by a sufficient increase of $\dot{M}$, and the existence of the disk tilt did not affect the condition of thermal stability throughout the disk. It might be possible that a weak tilt (with a possible signal of negative superhumps below the detection

limit) was present in 2018 when $\dot{M}$ somewhat decreased.

We must note that our observations were not as ideal as in KIC 9406652 and had long longitudinal observational gaps. We had only $6 \mathrm{hr}$ (at most) continuous runs each night with samplings in every $4-5 \mathrm{~min}$, and this observing condition was not ideal for detecting a weak signal of negative superhumps. A search for negative superhumps with a better temporal coverage and resolution when IM Eri is again in the IW And-type state, or in other objects, would solve the current discrepant result.

\section{Acknowledgments}

We acknowledge with thanks the variable star observations from the AAVSO International Database contributed by observers worldwide and used in this research. We are also grateful to the ASAS-3 and ASAS-SN teams for making the past photometric database available to the public. 


\section{Summary}

We made time-resolved photometry of IM Eri, which experienced the IW And-type state (a repeated sequence of standstill-like damping oscillation terminated by brightening followed by a dip) in 2018. We recorded three cycles and following the brightening in 2019 February. After these three cycles, the object gradually brightened to a standstill which lasted for more than $70 \mathrm{~d}$. We identified two periods during the IW And-type state: $4-5 \mathrm{~d}$ smallamplitude oscillations (often damping) and 34-43 d long cycle. We confirmed that these small-amplitude oscillations cannot be explained by the changing aspect of a precessing tilted disk. The states of small-amplitude oscillations were terminated by "terminal outbursts". The entire behavior is typical for an IW And-type object and the current observation provides the best confirmation of damping oscillations arising from the intrinsic variation of the disk luminosity. The object gradually brightened between these terminal outbursts. This finding agrees with the prediction of thermal-viscous disk instability in a tilted disk Kimura et al. (2019). We, however, did not succeed in detecting negative superhumps, which are considered to be the signature of a tilted disk.

\section{Supporting information}

Additional supporting information can be found in the online version of this article: E-table 1, E-figures 1,2. Supplementary data is available at PASJ Journal online.

\section{References}

\author{
Armstrong, E., et al. 2013, MNRAS, 435, 707 \\ Chen, A., O'Donoghue, D., Stobie, R. S., Kilkenny, D., \& Warner, B. \\ 2001, MNRAS, 325, 89 \\ Cleveland, W. S. 1979, J. Amer. Statist. Assoc., 74, 829 \\ Fernie, J. D. 1989, PASP, 101, 225 \\ Gies, D. R., et al. 2013, ApJ, 775, 64 \\ Hameury, J.-M., \& Lasota, J.-P. 2014, A\&A, 569, A48 \\ Kato, T. 2019, PASJ, 71, 20 \\ Kato, T., \& Maehara, H. 2013, PASJ, 65, 76 \\ Kato, T., et al. 2010, PASJ, 62, 1525 \\ Kato, T., \& Uemura, M. 2012, PASJ, 64, 122 \\ Kato, T., Uemura, M., Ishioka, R., Nogami, D., Kunjaya, C., Baba, \\ H., \& Yamaoka, H. 2004, PASJ, 56, S1 \\ Kimura, M., Osaki, Y., Kato, T., \& Mineshige, S. 2019, PASJ, \\ submitted \\ Kochanek, C. S., et al. 2017, PASP, 129, 104502 \\ Mason, E., \& Howell, S. B. 2016, A\&A, 589, A106 \\ Meyer, F., \& Meyer-Hofmeister, E. 1983, A\&A, 121, 29 \\ Montgomery, M. M. 2009, MNRAS, 394, 1897 \\ Osaki, Y. 1996, PASP, 108, 39 \\ Pojmański, G. 2002, Acta Astron., 52, 397
}

Shappee, B. J., et al. 2014, ApJ, 788, 48

Simonsen, M. 2011, J. American Assoc. Variable Star Obs., 39, 66

Simonsen, M., Bohlsen, T., Hambsch, F.-J., \& Stubbings, R. 2014, J.

American Assoc. Variable Star Obs., 42, 199

Stellingwerf, R. F. 1978, ApJ, 224, 953

Szkody, P., et al. 2013, PASP, 125, 1421

Tibshirani, R. 1996, J. R. Statistical Soc. Ser. B, 58, 267

Warner, B. 1995, Cataclysmic Variable Stars (Cambridge:

Cambridge University Press)

Wood, M. A., \& Burke, C. J. 2007, ApJ, 661, 1042 